\title{
Argos, fouilles de l'Aspis - 2017, 2018
}

Anna Philippa-Touchais, Gilles Touchais, Natalie Abell, Anthi Balitsari, Maria Choleva et Julie Hruby

\section{OpenEdition \\ Journals}

Édition électronique

URL : http://journals.openedition.org/baefe/1752

DOI : $10.4000 /$ baefe. 1752

ISSN : 2732-687X

Éditeur

ResEFE

\section{Référence électronique}

Anna Philippa-Touchais, Gilles Touchais, Natalie Abell, Anthi Balitsari, Maria Choleva et Julie Hruby, «Argos, fouilles de l'Aspis - 2017, 2018 » [notice archéologique], Bulletin archéologique des Écoles françaises à l'étranger [En ligne], Grèce, mis en ligne le 26 novembre 2020, consulté le 22 mars 2021. URL : http://journals.openedition.org/baefe/1752 ; DOI : https://doi.org/10.4000/baefe.1752

Ce document a été généré automatiquement le 22 mars 2021

\section{cc) $(1)$}

Le Bulletin archéologique des Écoles françaises à l'étranger est mise à disposition selon les termes de la Licence Creative Commons Attribution - Pas d'Utilisation Commerciale - Pas de Modification 4.0 International. 


\title{
Argos, fouilles de l'Aspis - 2017, 2018
}

\author{
Anna Philippa-Touchais, Gilles Touchais, Natalie Abell, Anthi Balitsari, \\ Maria Choleva et Julie Hruby
}

\section{NOTE DE L'AUTEUR}

Autorité nationale présente : Éphorie des antiquités d'Argolide Numéro de mission : B05

Composition de l'équipe : Natalie Abell (Prof., Université de Michigan), Anthi Balitsari (post-doctorante, Université de Louvain-la-Neuve), Maria Choleva (post-doctorante, Fondation Fyssen), Oreste Decavallas (chercheur indépendant), Armelle Gardeisen (IR, UMR 5140), Julie Hruby (Prof., Bryn Mawr College), Maria Kayafa (Dr., Centre d'Éducation Environnementale, Lavrion), Evangelia Kiriatzi (Director, Fitch Laboratory, BSA), Evi Margariti (Prof., The Cyprus Institute), Odysseas Metaxas (chercheur indépendant ), Yannis Nakas (dessinateur), Nikos Sepetzoglou (dessinateur), Vivian Philippa (architecte), Anna Philippa-Touchais (Chercheur associée, EFA, UMR 7041), Gilles Touchais (Prof. Université Paris 1, UMR 7041 ArScAn), Sevi Triantaphyllou (Prof., Université de Thessalonique), Efi Tsiolaki (doctorante, Université de Cincinnati).

Établissements porteurs de l'opération : EfA

\section{Données scientifiques produites :}

Argos par l'EFA

1 Les années 2017 et 2018 ont été consacrées, d'une part, à l'écriture du premier volume de la publication finale des fouilles de l'Aspis, qui a été remis au Service des publications de l'EfA à l'été $2020^{1}$; d'autre part, à l'étude de deux ensembles de matériel, qui seront publiés ultérieurement $: 1$ ) la céramique mésohelladique issue des fouilles récentes, dont l'étude sera incluse dans le second volume de la publication finale $e^{2}$ 2) le matériel votif archaïque des fouilles Vollgraff, localisé récemment au musée d'Argos ${ }^{3}$ et qui fera l'objet d'une publication séparée. Ajoutons que la campagne de 2017 a été complétée par des travaux de consolidation dans le secteur Nord du site. 


\section{Étude de la céramique HM}

2 L'étude typologique et contextuelle des assemblages céramiques liés à la maison MI (secteur Nord), qui avait été entreprise en $2015^{4}$, a été achevée par A. Philippa-Touchais en collaboration avec A. Balitsari, qui a aussi complété le dossier graphique de ce matériel. D'autre part, M. Choleva a poursuivi l'étude des techniques de façonnage mises en œuvre au sein des mêmes assemblages. Enfin, N. Abell a entrepris un examen macroscopique poussé des deux grandes classes de céramique à décor peint : céramique à peinture mate $(\mathrm{PM})$ et céramique à peinture lustrée $(\mathrm{PL})$.

\section{Les techniques de façonnage}

Maria Choleva

La poursuite de l'étude technologique de la céramique issue de la maison MI, datée de l'HM II, a confirmé, d'une part, le faible taux de récipients façonnés au tour $(8 \% \mathrm{du}$ matériel conservé), le reste de la production étant réalisé par des techniques manuelles (colombins, plaques) ; d'autre part, le lien privilégié entre la technique de l'élaboration au tour et la céramique grise fine polie (minyenne), surtout pour la fabrication des canthares et des bols renflés, moins souvent pour celle des jattes. La technique de l'élaboration au tour est au contraire rarement utilisée pour la fabrication des vases décorés à la peinture mate, et seulement de façon très marginale pour celle des récipients à peinture lustrée. L'étude comparative de la céramique de la «maison fantôme" (secteur Sud-Est), datée de la fin de l'HM I, livre une image un peu différente : tous les pots élaborés au tour sont en minyen gris et leur taux au sein de l'assemblage est plus élevé $(20 \%)$, le type le plus fréquent étant le petit bol pansu à deux anses.

\section{Analyse macroscopique des céramiques à décor peint}

Natalie Abell

4 L'étude a porté, d'une part, sur la céramique de la maison MI ; d'autre part sur une large sélection de tessons à peinture mate $(\mathrm{PM})$ et à peinture lustrée $(\mathrm{PL})$ issus des fouilles du secteur Nord.

5 L'examen du matériel de la maison MI a permis de repérer d'autres fragments de céramique à peinture mate d'Égine, de Minyen gris (avec diverses variantes) et de séries potentiellement locales. Dans les quelques fragments de céramique grossière examinés on a relevé la présence de grains de quartz, d'inclusions calcaires (noires, rouges, brunes) angulaires ou subangulaires, ainsi que de rares paillettes de mica argenté. Les inclusions présentes dans la céramique sombre lissée - présumée locale sont le quartz et des particules de roches sombres (sédimentaires ?) qui pourraient être de même nature que celles de la céramique grossière.

6 L'étude des céramiques à décor peint du secteur Nord avait pour objectifs 1) de caractériser les matières premières locales et importées ; 2) de définir des groupes macroscopiques, qui seront ensuite testés par des analyses pétrographiques et chimiques; 3) de déterminer les marqueurs techniques des productions locales et 
importées, afin de reconstituer les chaines opératoires associées aux unes et aux autres et d'évaluer les interactions entre les communautés de pratique locales et exogènes.

$7 \quad$ L'étude a permis de distinguer deux grands groupes macroscopiques : l'un (A), présumé local, est caractérisé par une pâte relativement bien cuite avec inclusions de roches sédimentaires et parfois une fine fraction de mica (surtout argenté), sans trace de volcanisme; l'autre (B) par une pâte tendre et poreuse, des inclusions probablement volcaniques et du mica relativement fréquent. Le groupe $A$ a été subdivisé en neuf sousgroupes (A1 à A9) (fig. 1-2), le groupe B en cinq sous-groupes (B1 à B5) (fig. 3-4), dont certains pourraient être des variantes à l'intérieur d'une même source: les sousgroupes B1, B2 et B3, par exemple, ont toute chance de provenir tous les trois d'Égine. À ces deux grands groupes il faut en ajouter deux autres, minoritaires, dont chacun a été subdivisé en deux sous-groupes : le groupe $\mathrm{C}$, caractérisé par une matrice probablement micacée et des inclusions métamorphiques (fig. 5), et le groupe $\mathrm{F}$ par sa texture fine et la rareté des inclusions (fig. 6). La plupart des pâtes associées à la céramique à peinture lustrée (A7-A9) contiennent des inclusions très similaires, mais rien ne permet encore d'affirmer qu'elles proviennent de la même source. On a également identifié quelques importations de Mélos/Théra (B5).

Fig. 1. Groupe A1 : a) surface, b) cassure.
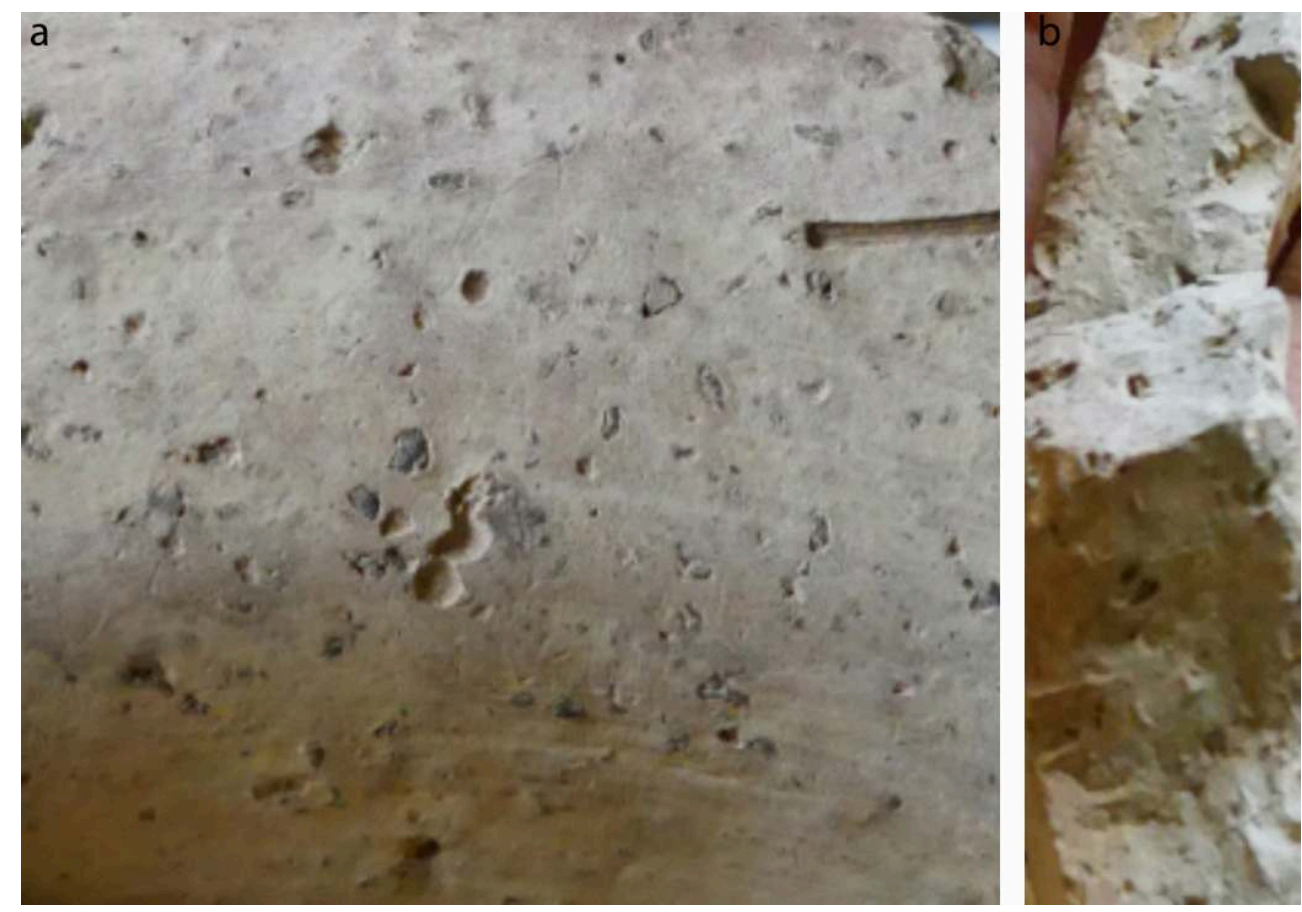
Fig. 2. Groupe A3 : a) surface, b) cassure.
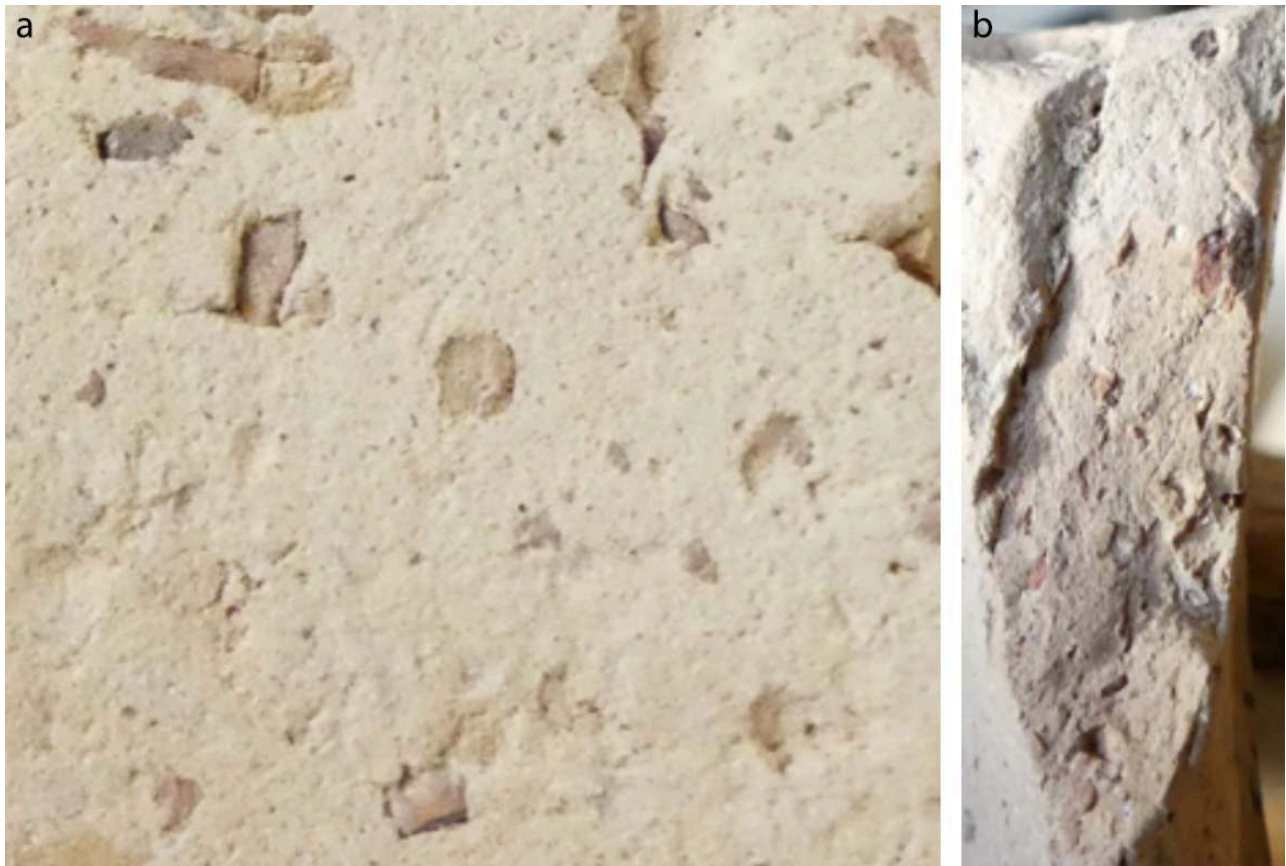

EFA.

Fig. 3. Groupe B2 : a) surface, b) cassure.
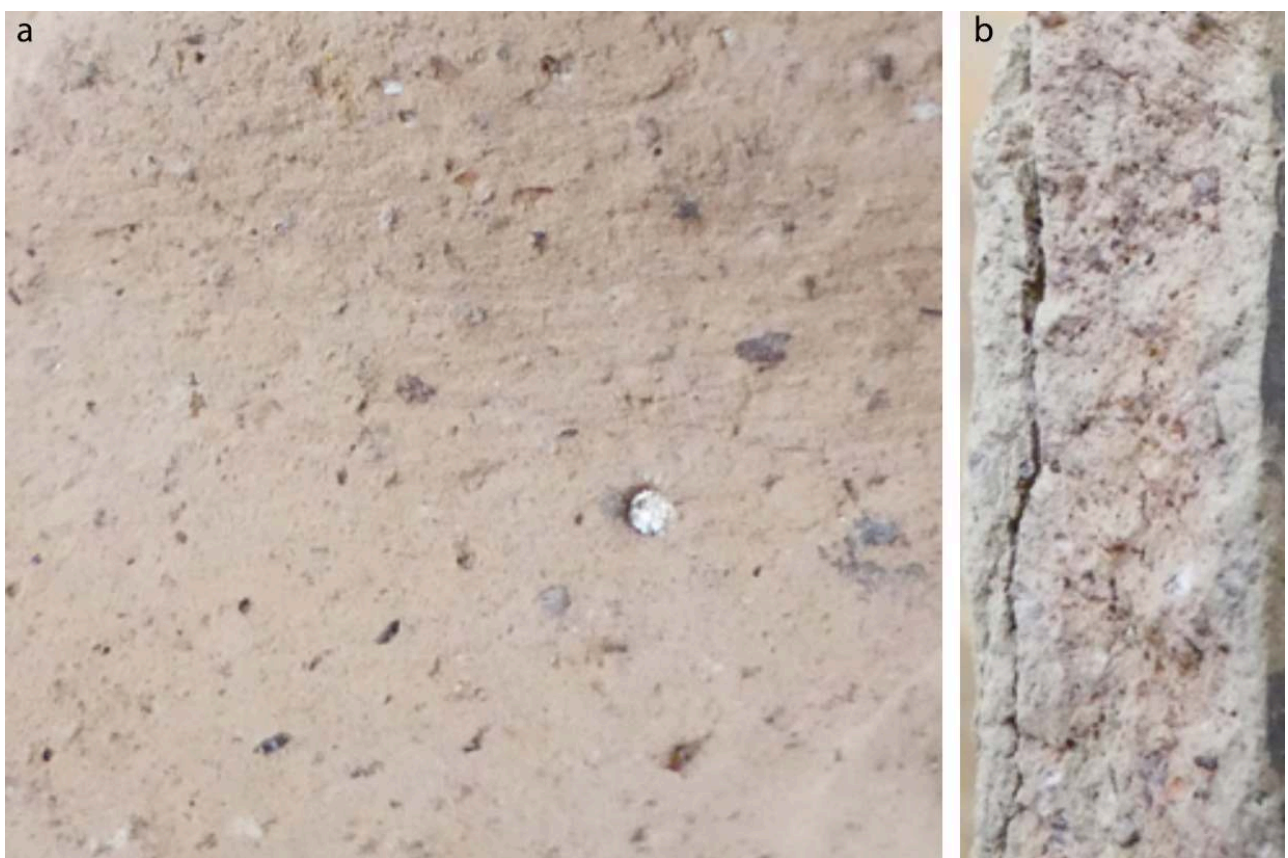

EFA. 
Fig. 4. Groupe B4 : a) surface, b) cassure.
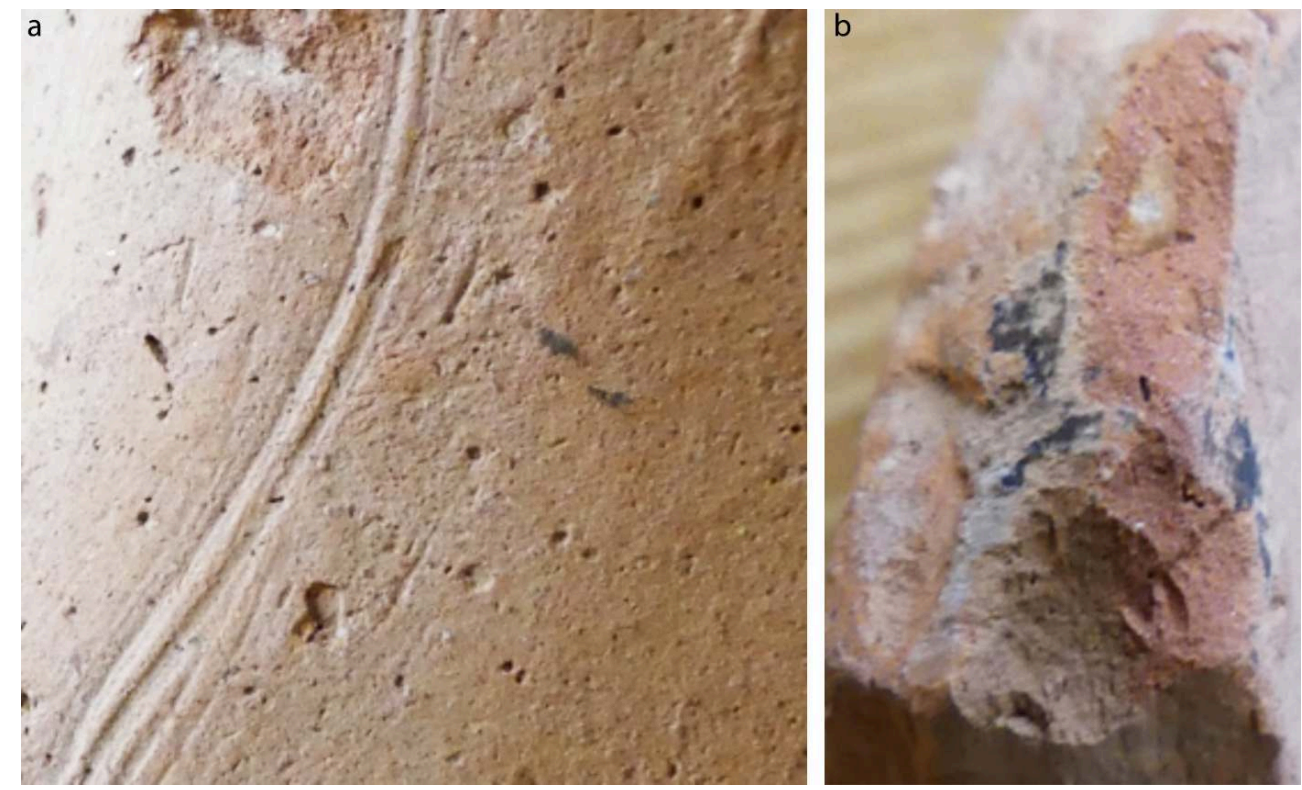

EFA.

Fig. 5. Groupe $C 1$ : a) surface, b) cassure.
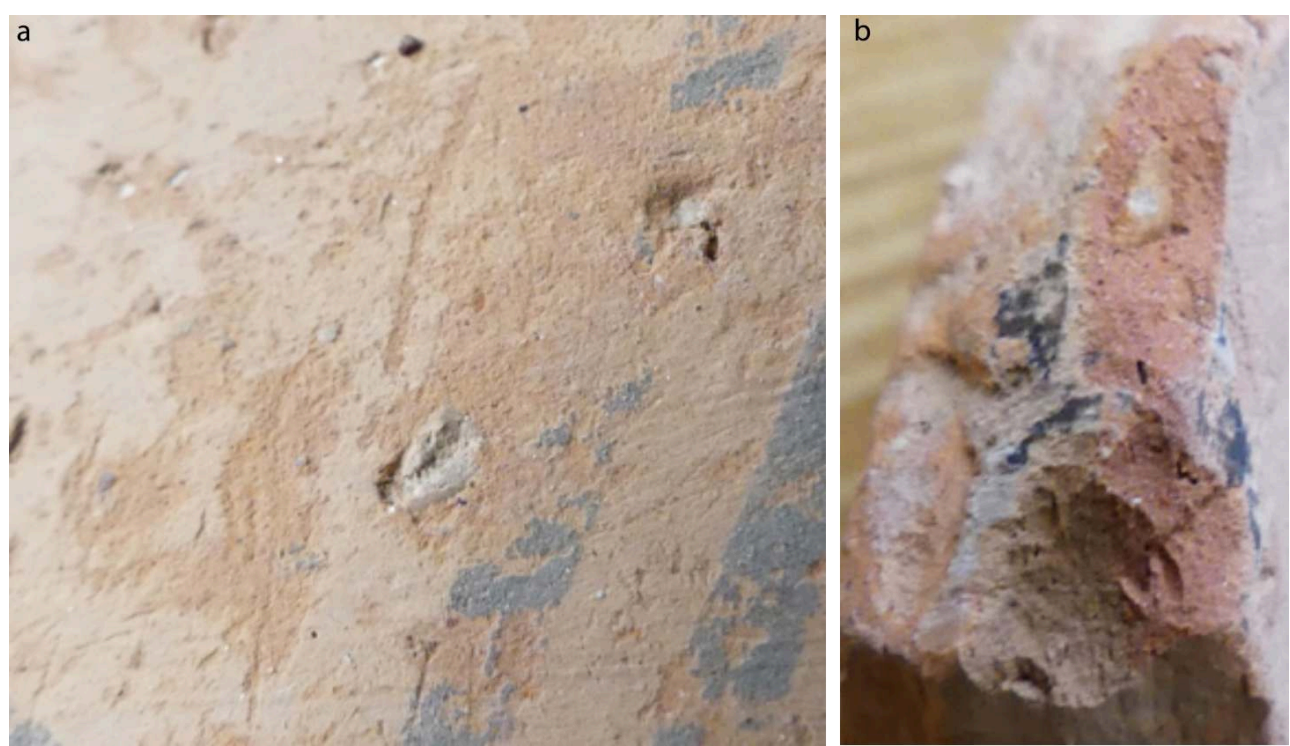

EFA. 
Fig. 6. Groupe B4 : a) surface, b) cassure.
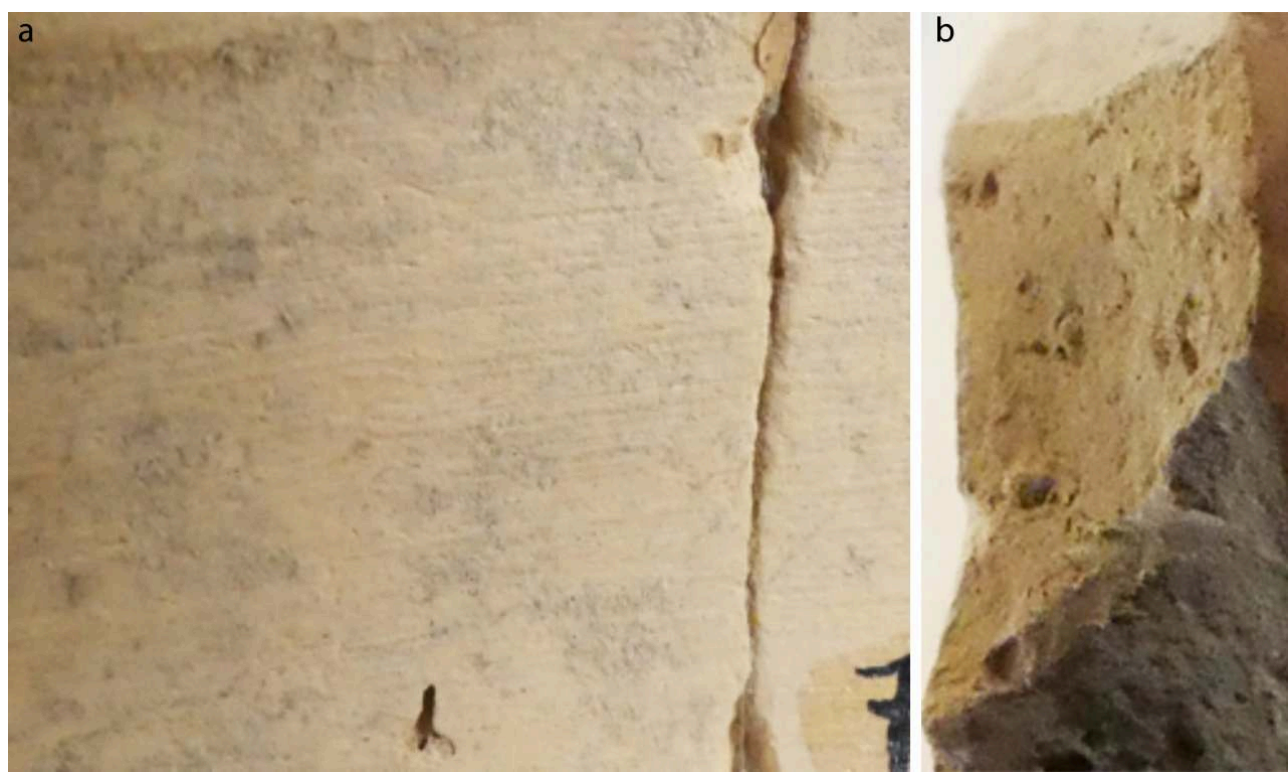

EFA.

$\mathrm{Du}$ point de vue technologique, bien qu'il soit encore trop tôt pour proposer des interprétations en matière de communautés de pratique, on observe toutefois certaines constantes, notamment au niveau de l'engobage : la plupart des tessons du groupe A1 présentent des traces plus ou moins nettes d'engobe (ou d'«auto-engobe»), à la différence de ceux du groupe $B$ dans lequel elles sont très rares. En recvanche, la variabilité est beaucoup plus grande, à l'intérieur des groupes, en ce qui concerne les techniques d'égalisation des colombins ou de fixation des anses, la qualité de la peinture et la couleur, pour lesquelles d'autres paramètres (forme des vases, classe céramique) entrent sans doute en ligne de compte.

\section{Étude du matériel votif archaïque}

\section{Le matériel votif des fouilles Vollgraff}

Repéré lors des travaux préparatoires au déménagement de la réserve des fouilles françaises, le matériel archaïque des premières fouilles de l'Aspis se composait de deux ensembles : d'une part, un lot d'objets appartenant au dépôt votif archaïque découvert au sommet de la colline "à $25 \mathrm{~m}$ au Sud de l'église du Prophète Élie »", d'autre part, un certain nombre de figurines et autres ex-voto susceptibles, d'après certains indices, de provenir aussi des fouilles de Vollgraff sur l'Aspis.

Le matériel du dépôt votif fouillé par Vollgraff se trouvait réparti dans trois tiroirs différents dont un seul portait une indication de provenance; le contenu des deux autres a été identifié grâce à des recollages ou à des descriptions d'objets laissées par le fouilleur. Le premier tiroir (E1) contient environ 55 pièces - céramique et objets miniatures -, le deuxième (E81) 58 pièces - surtout des figurines (fig. 7), dont la tête dite "d'Apollon» (fig. 8) mentionnée par Vollgraff ${ }^{6}$ - et le troisième (D97/W23) environ 90 pièces - surtout des vases miniatures (fig. 9) et des fragments de céramique corinthienne, dont un couvercle de pyxide (fig. 10) correspondant à une description de 
Vollgraff 7 , et aussi quelques objets miniatures (couronnes, grenades) -, soit un total d'environ 200 pièces. Un quatrième tiroir (E103) contient 78 ex-voto - essentiellement des figurines - qui ont des chances de provenir aussi de l'Aspis. La majorité de ces objets date de l'époque archaïque, mais il y en a aussi un certain nombre - surtout de la céramique et quelques figurines - d'époque classique et hellénistique (fig. 11).

Fig. 7. Figurines archaïques.

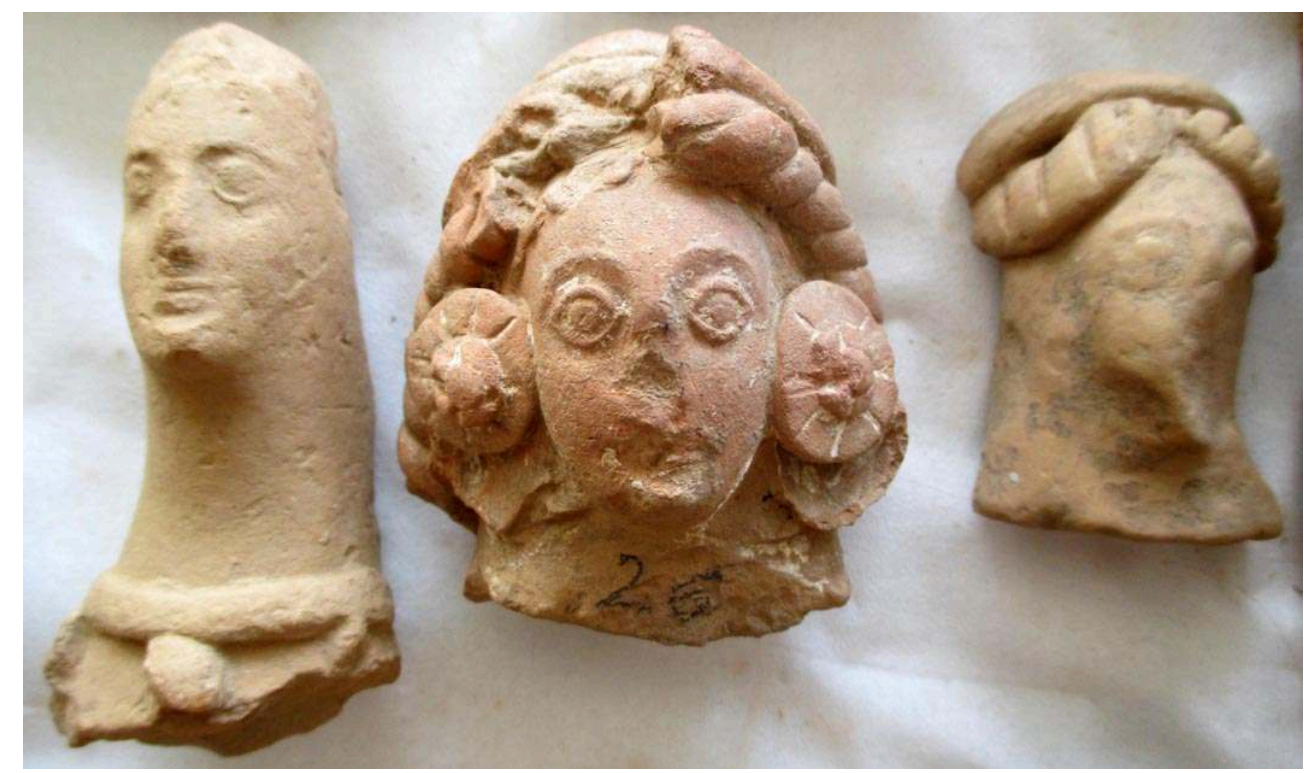

EFA.

Fig. 8. Tête d'« Apollon » (1902/85.3).

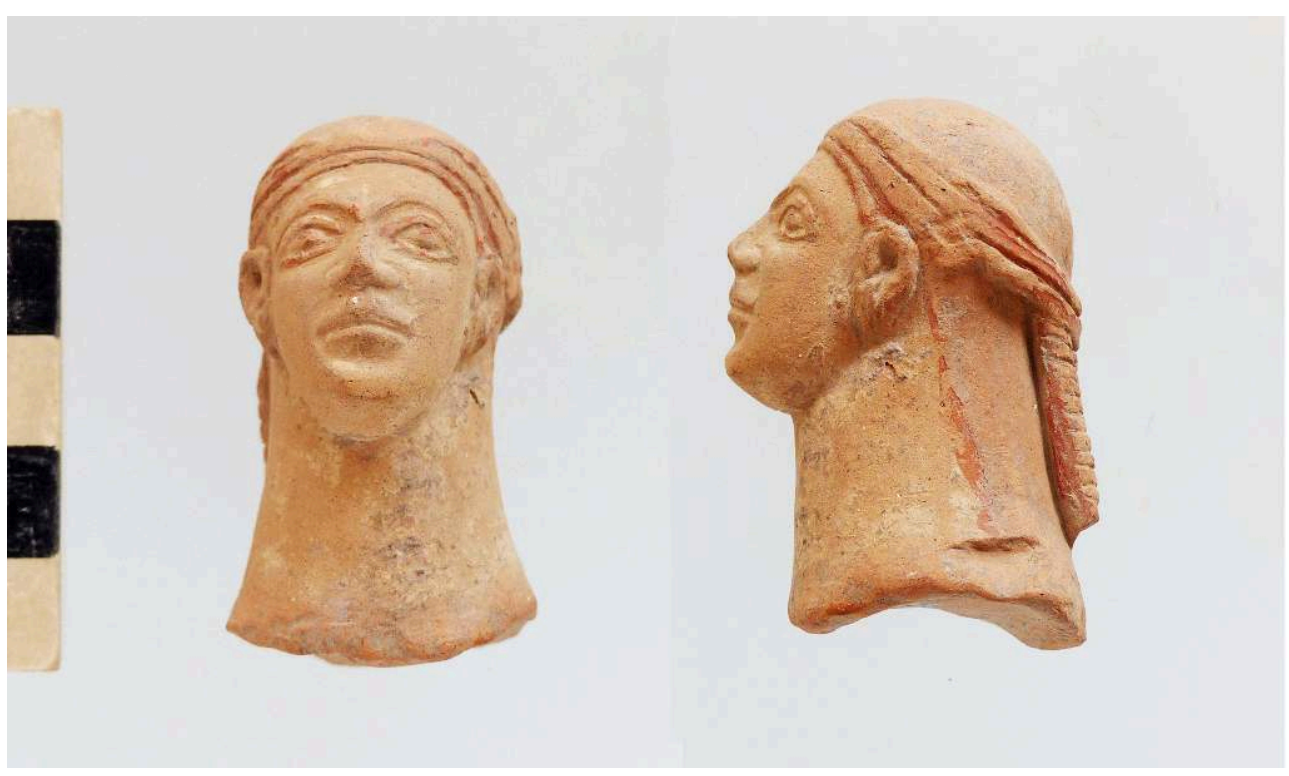

EFA. 
Fig. 9. Vases miniatures (fin $\mathrm{VIII}^{\mathrm{e}}-\mathrm{VII}{ }^{\mathrm{e}}$ s. av. J.-C.).

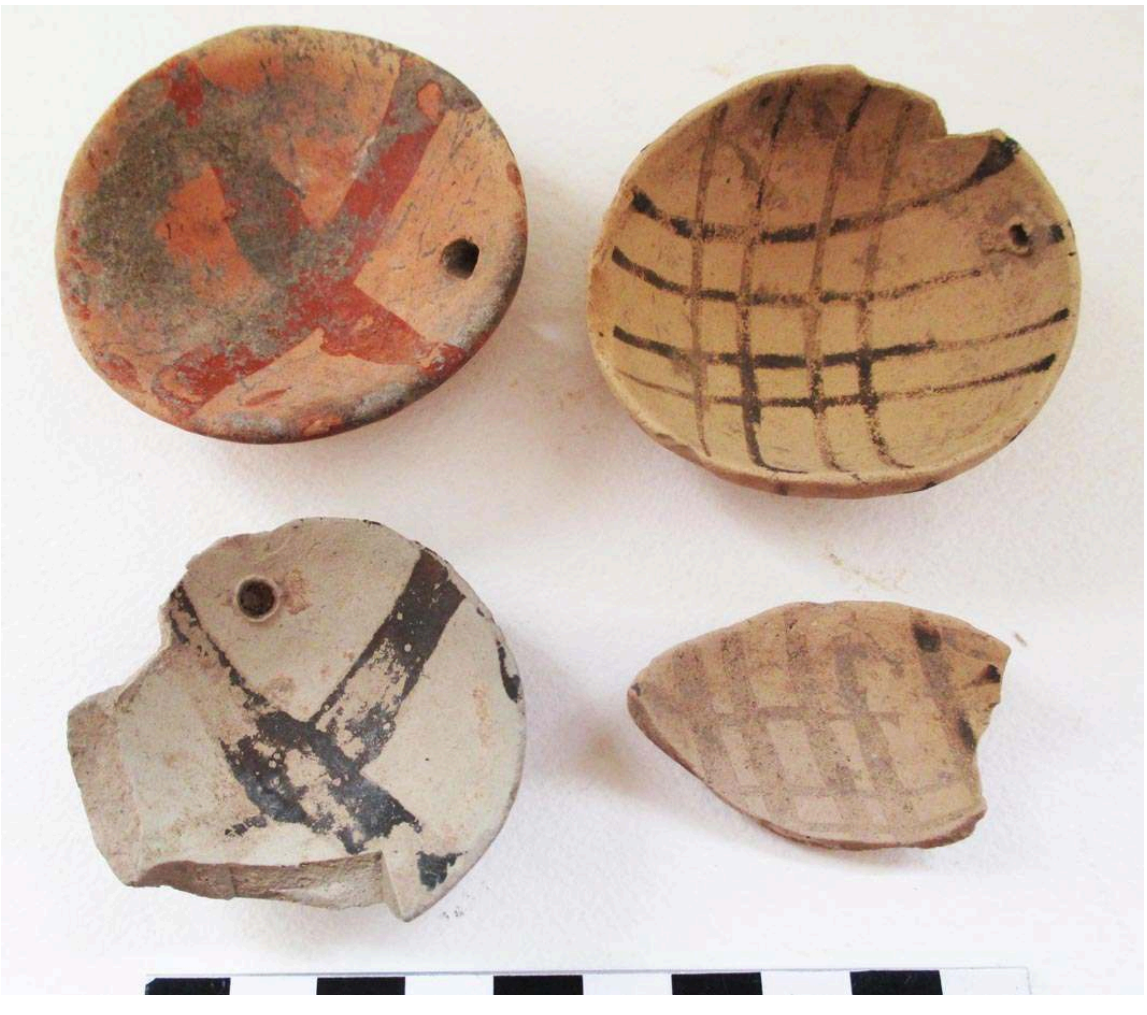

EFA.

Fig. 10. Couvercle de pyxide corinthienne (1902/85.6).

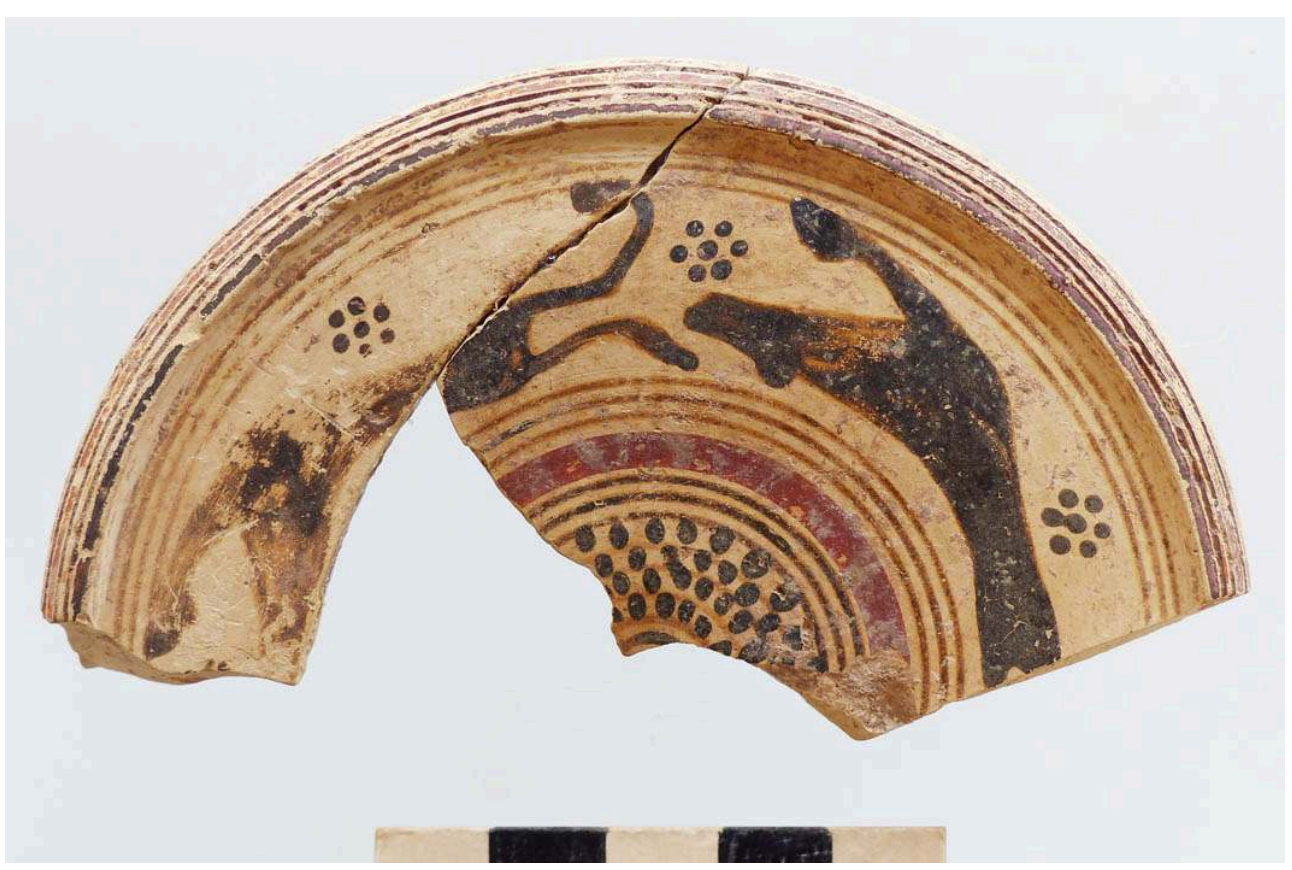

EFA. 
Fig. 11. Figurines hellénistiques.

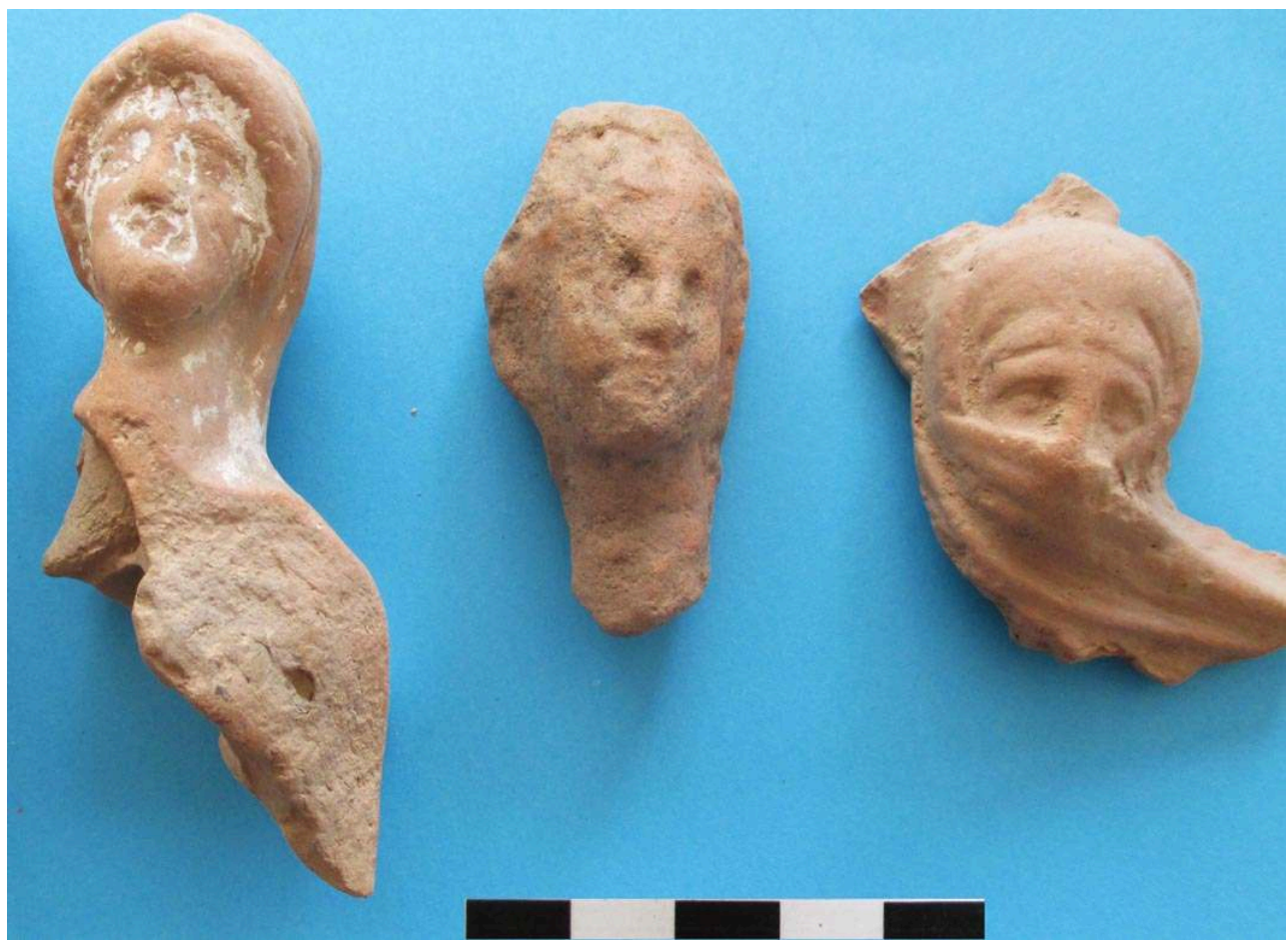

EFA.

En 2018 une partie du matériel été nettoyée et restaurée par A. Konstantatos, restaurateur de l'EfA. Une vingtaine d'entre elles ont ensuite été dessinées par N. Sepetzoglou (fig. 12-14) et enregistrées dans une base de données.

Fig. 12. Figurine archaïque (1902/103.7).

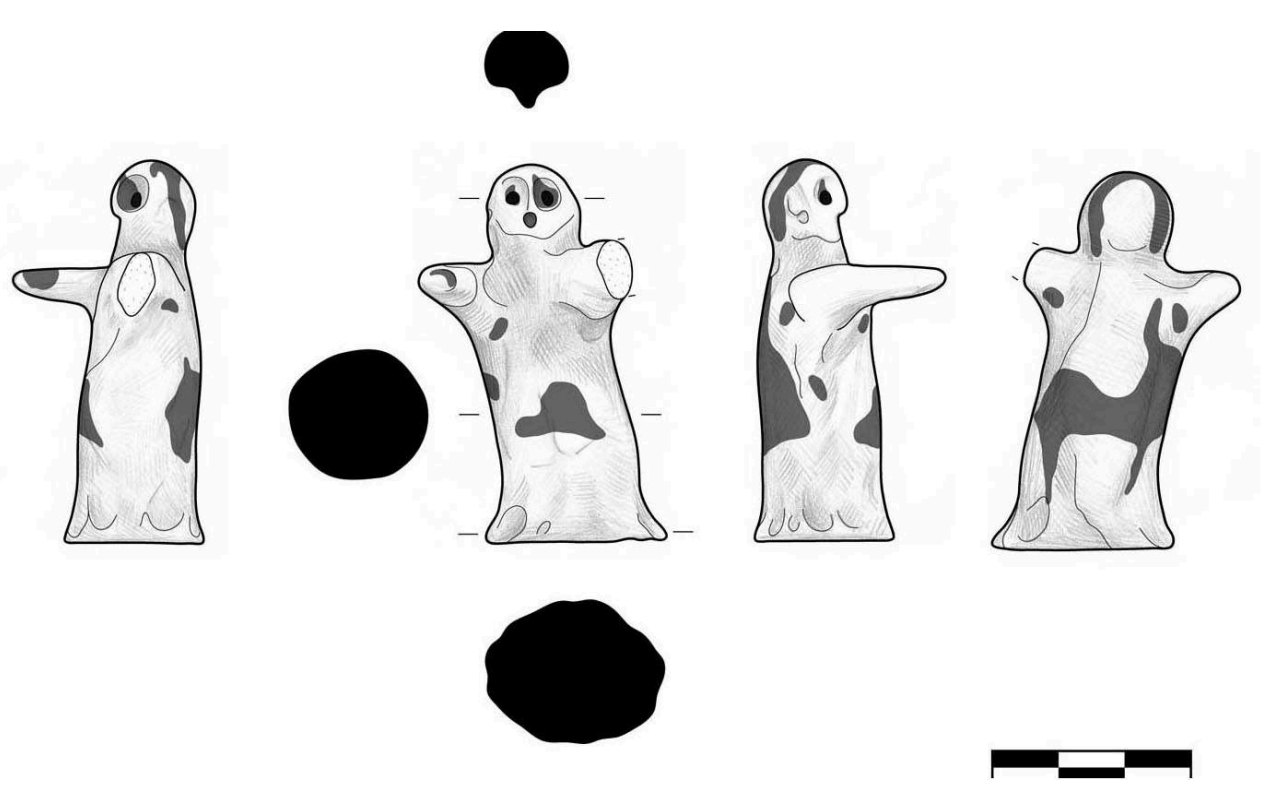

EFA, dessin N. Sepetzoglou. 
Fig. 13. Figurine de cheval (1902/103.11).

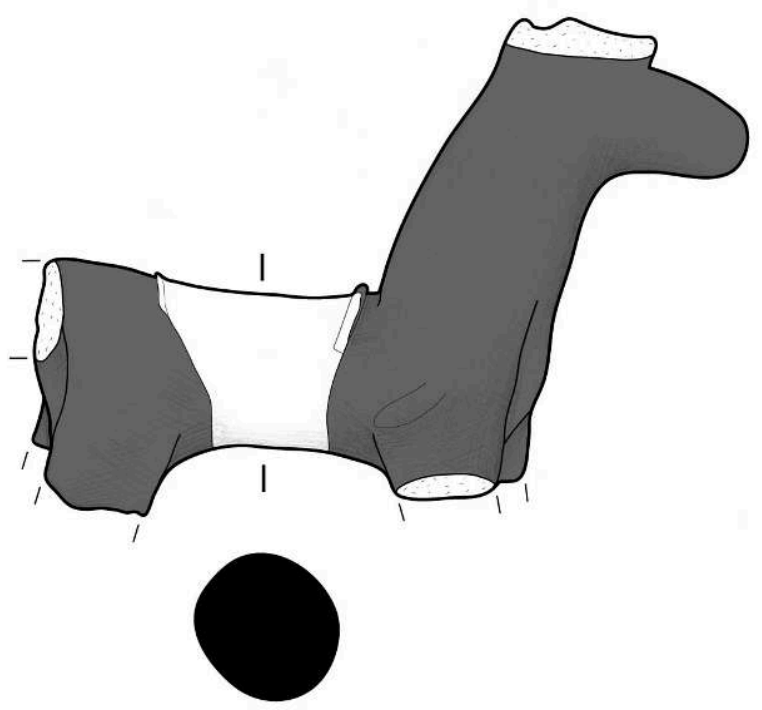

EFA, dessin N. Sepetzoglou.

Fig. 14. Coupelle archaïque (1902/85.4).

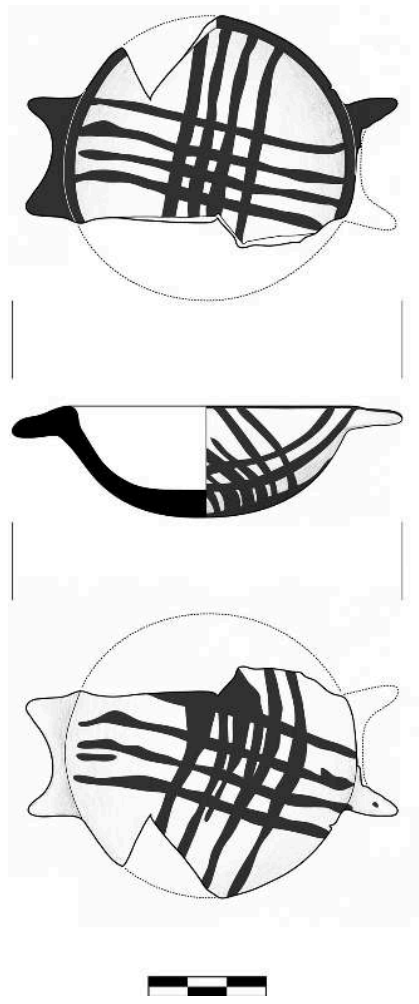

EFA, dessin N. Sepetzoglou.
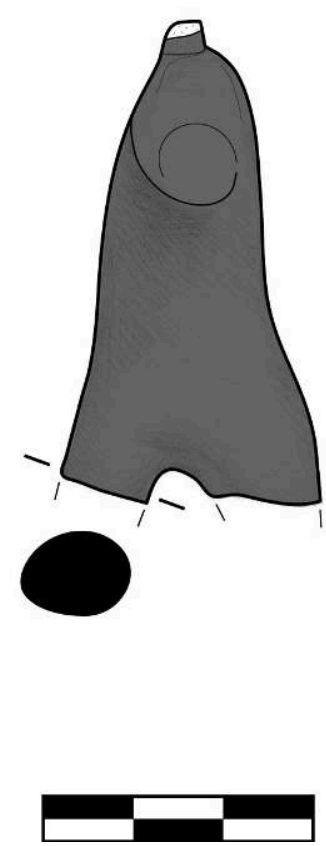


\section{Étude des empreintes digitales}

12 Dans le cadre de l'étude du matériel votif archaïque de l'Aspis, on a jugé intéressant de procéder à l'examen macroscopique des empreintes digitales relevées sur un certain nombre de vases, figurines et autres objets (fig. 15) dont quelques-uns seulement proviennent des fouilles Vollgraff, les autres ayant été trouvés lors des fouilles récentes. Ce travail a été confié à J. Hruby (Darmouth College), spécialiste de la question, qui a opéré avec l'assistance de D. Karali (fig. 16) et dont nous reproduisons ci-dessous le rapport :

13 " The primary goal of the 2018 study of fingerprints from the site of Argos was to identify the sexes and approximate ages of the producers of ceramics, including figurines and diminutive vessels, from the site during the Late Geometric, Archaic and Classical periods. Establishing both ages and sexes will contribute to our understanding of the ways in which ancient Greeks engaged in cult practice and in conceptualizing issues like gender, apprenticeship, and division of labor. Concrete archaeological evidence for the identity of ceramics producers from a site that is outside Athens will make it possible to problematize modern Athenocentric discussions of gender and of labor. An additional benefit is that if the producers of the figurines and miniature vessels that come from a single, major ritual deposit (79/363) are discovered to have been female, this would provide some additional indication that the assemblage from which they came may have honored a female divinity. Regardless of what the sex or age of the producers are (and it appears that many are juveniles), we will also be obligated to think about the relationship between the producer and the dedicant.

Our process was as follows. First, prints were selected, with criteria including the size of the print area and the clarity of the print; when multiple prints crossed each other in large part, or when prints had been preserved but the clay had been wet when they were formed, or when prints had been smudged, those were not included in the sample. Among the types of objects from which prints were scanned were figurines of furniture, wreaths, animals, and human figures, in addition to miniature, handmade bowls; the latter were often but not always very simply painted. Prints were scanned from 36 objects. Many of these had more than one print preserved, and for each artifact, we scanned as many prints as we believed we might be able to get good results from. Each print was 3D scanned using a Keyence VR-3100 structured light scanner. All were scanned at 12x and 50x resolutions; when it was possible to see ridge detail at 160x resolution, scans with limited dimensions were also made at that resolution.

Based on preliminary study, there appear to have been a substantial number of young producers of both bowls and figurines, which would provide an example supporting Susan Langdon's $2013^{8}$ interpretation of the diminutive vessels found at several different sites in the Argolid as having been made by worshippers and their children ( $\mathrm{p}$. 179).

16 A more rigorous methodology for sexing and aging print scans is still under development by our team's computer scientists, but our initial, exploratory analysis will utilize simpler techniques that have been used in archaeological contexts in the past. These include an analysis of the distribution of mean ridge breadths (which correlate with both sex and age) and an evaluation, where possible, of the shape of the margins of the dermal ridges; when dermal ridges are shaped more like beads on strings than like more consistent bands, they typically indicate juvenile age grades. » 
Fig. 15. « Fleur double » avec empreinte digitale (79/363.68).

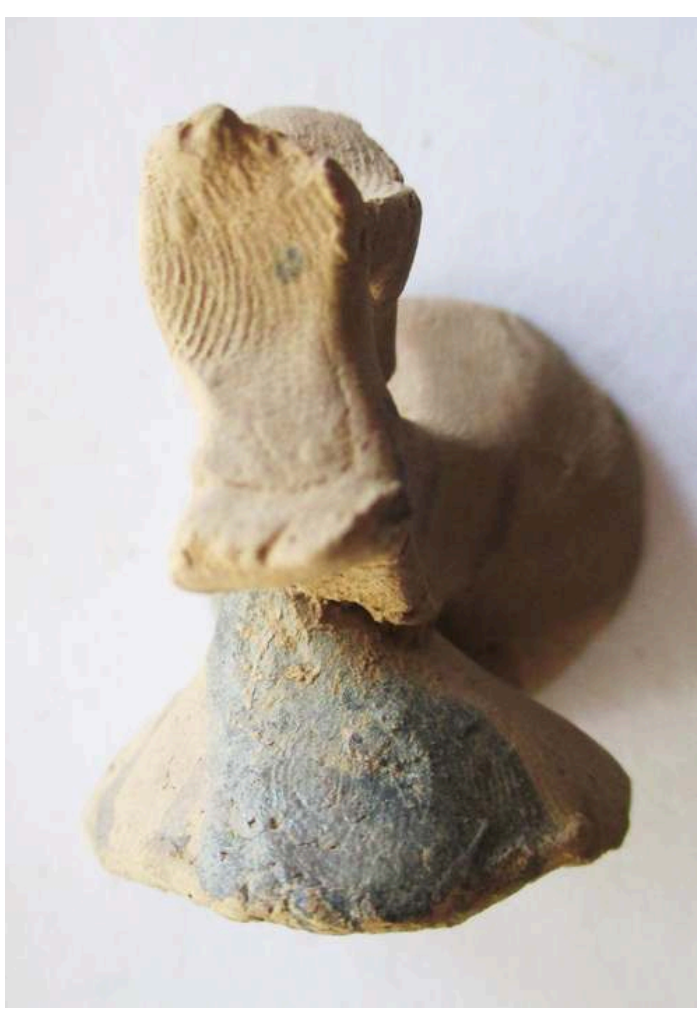

EFA.

Fig. 16. J. Hruby et D. Karali examinant les empreintes digitales au musée d'Argos.

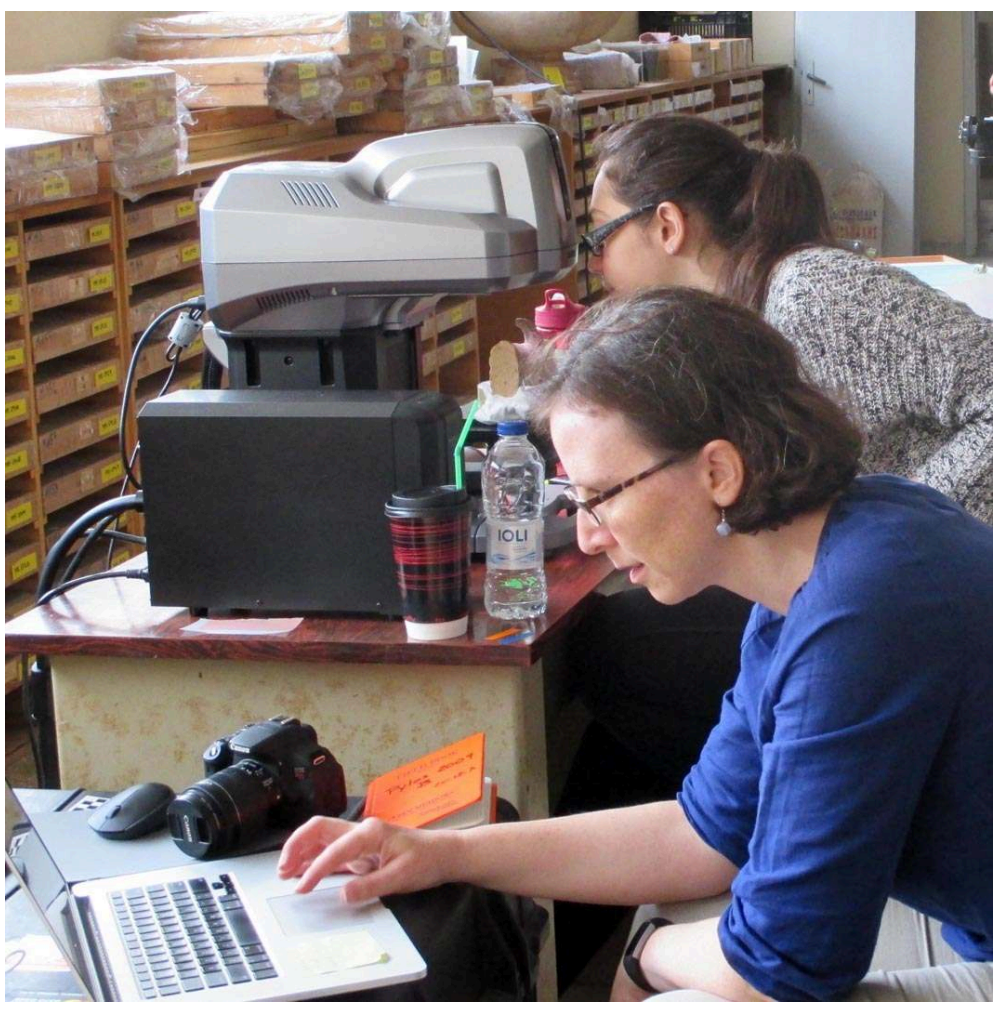

EFA. 


\section{Travaux de consolidation}

Les travaux de maintenance effectués sur le site en 2017 ont consisté, d'une part, à remonter le petit segment du parement intérieur du mur Est de la maison MI dégagé en 2010-2011 (mur 751) car, faute d'avoir été consolidé à temps, il s'était effondré (fig. 17, 1) ; d'autre part, à remettre en place un gros bloc du parement interne du rempart mésohelladique qui s'était effondré dans la fouille, travail d'autant plus important que ce bloc appartient à la seule section conservée de ce parement (fig. 17, 2).

Fig. 17. Aspis, plan du secteur Nord.

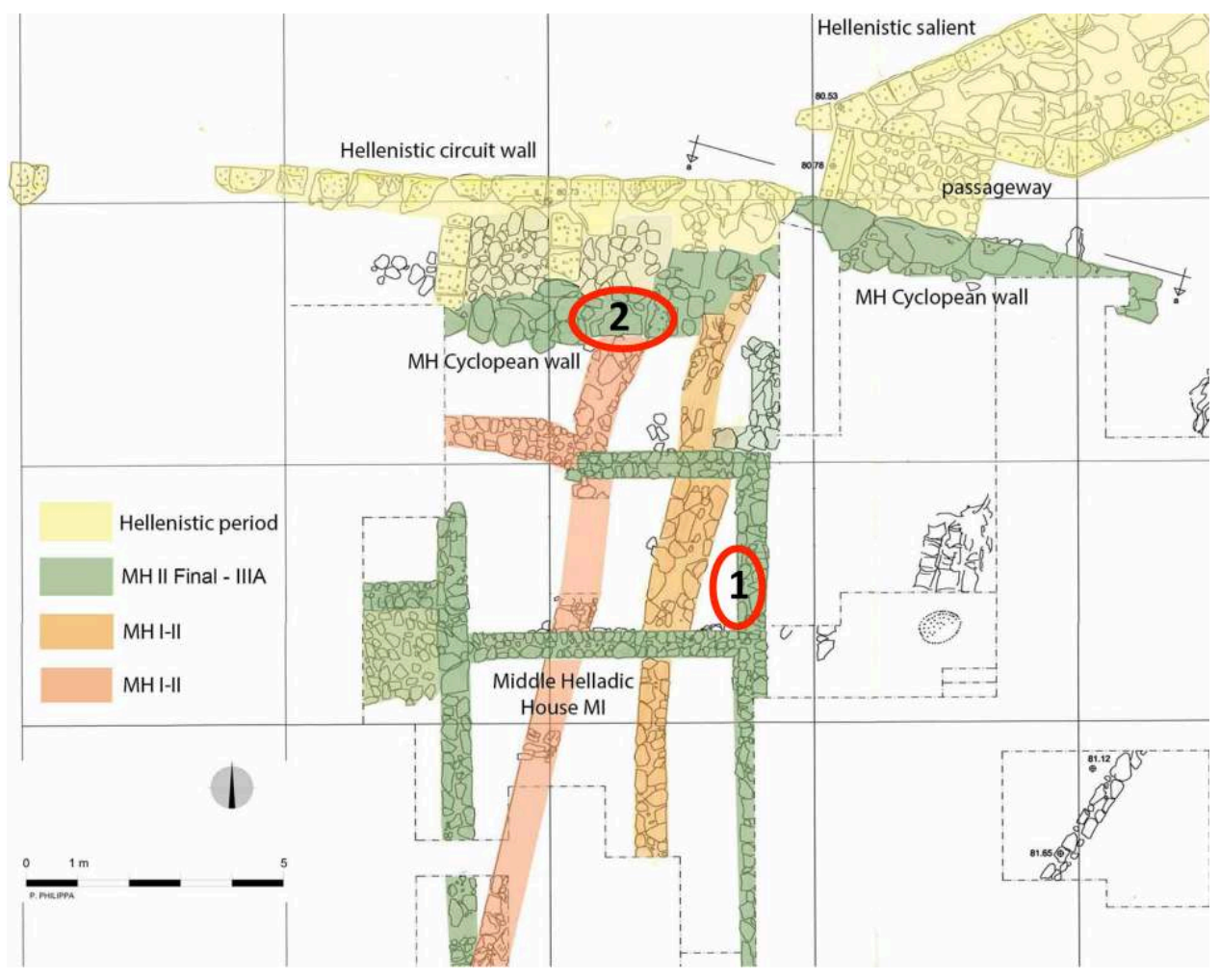

EFA.

Les pierres écroulées du mur 751, qui gisaient sur le sol de la maison MI (fig. 18), ont pu être remises en place sans grande difficulté, puis maçonnées et jointoyées (fig. 19). Un peu plus délicat a été le remontage du gros bloc du rempart, qui s'était brisé en plusieurs morceaux dans sa chute. Il a cependant pu être reconstitué, remis en place et fermement consolidé au mortier de chaux (fig. 20-21). 
Fig. 18-19. Le mur 751 avant et après les travaux de consolidation.

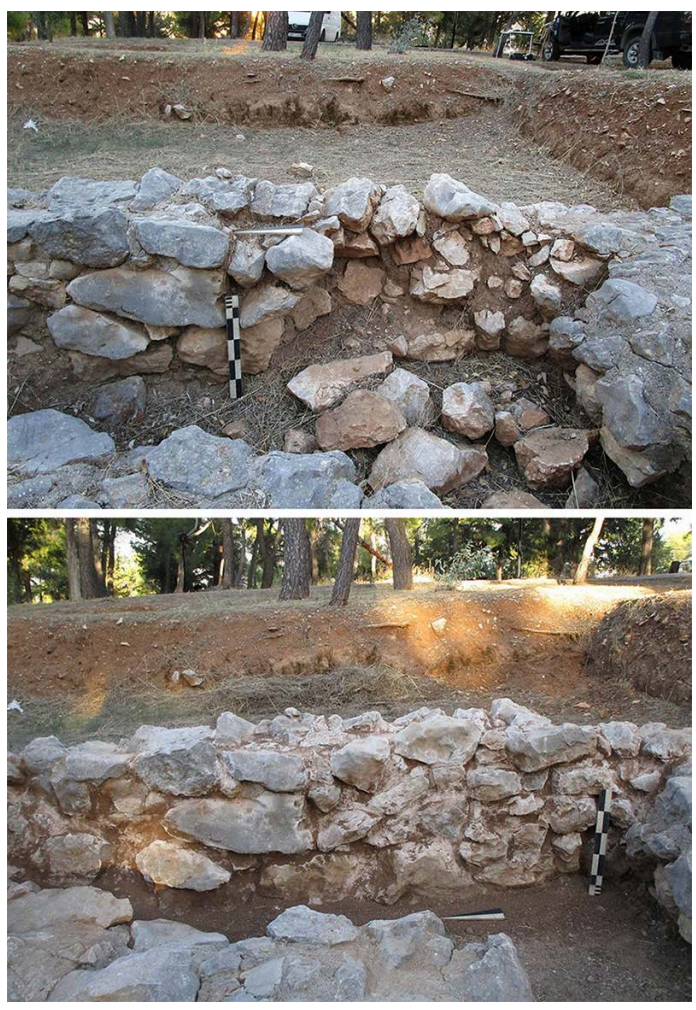

EFA.

Fig. 20-21. Le parement intérieur du rempart mésohelladique avant et après les travaux de consolidation.
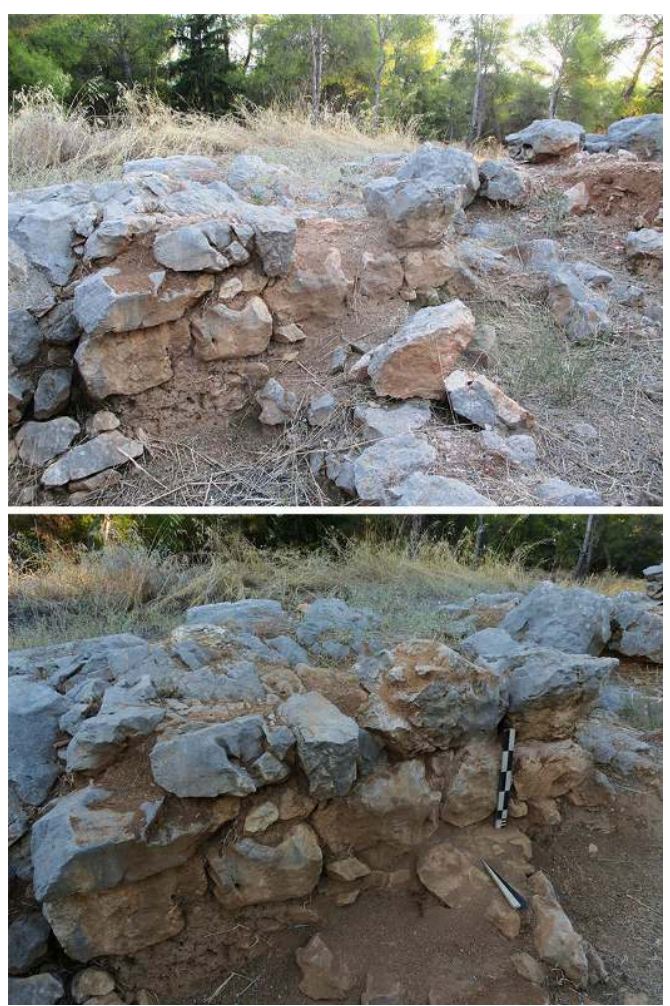

EFA. 


\section{Bibliographie}

19

Anna Philippa-Touchais, «Aperçu des céramiques mésohelladiques à décor peint de l'Aspis d'Argos, I: la céramique à peinture mate ", $B C H$ 126, 2002, p. 1-40.

Anna Philippa-Touchais, «Aperçu des céramiques mésohelladiques à décor peint de l'Aspis d'Argos, II: la céramique à peinture lustrée », BCH 127, 2003, p. 1-47.

Anna Philippa-Touchais, "Aeginetan matt-painted pottery at Middle Helladic Aspis, Argos ", in Florens Felten, Walter Gauss, Rudolfine Smetana (éd.), Middle Helladic Pottery and Synchronisms, Vienne, Verlag der Österreichischen Akademie der Wissenschaften, 2007, p. 97-113.

Anna Philippa-Touchais, «Settlement planning and social organisation in Middle Helladic Greece ", in Anna Philippa-Touchais et alii (éd.), Mesohelladika. La Grèce continentale au Bronze Moyen, Athènes, EFA, BCH Suppl. 52, 2010, p. 781-801.

Anna Philippa-Touchais, “'Cycles of Collapse in Greek Prehistory’: Reassessing social change at the beginning of the Middle Helladic and the Early Iron Age », in William D. E. Coulon, Alexander Mazarakis Ainian (éd.), The "Dark Ages" Revisited. Acts of an International Symposium in Memory of William D.E. Coulson, University of Thessaly, Volos, 14-17 June 2007, Volos, UP, 2011, p. 31-44.

Anna Philippa-Touchais, « Les tombes intra-muros de l'Helladique Moyen à la lumière des fouilles de l'Aspis d'Argos ", in Dominique Mulliez (éd.), Sur les pas de Wilhelm Vollgraff. Cent ans d'activités archéologiques à Argos, Athènes, EFA, Recherches francohelléniques 4, 2013, p. 75-100.

Anna Philippa-Touchais, "The Middle Bronze Age fortifications on the Aspis hill at Argos ", in Rune Frederiksen, Silke Müth, Peter I. Schneider, Mike Schnelle (éds), Focus on Fortifications: New Research on Fortifications in the Ancient Mediterranean and the Near East, Oxford, Oxbows Books, Fokus Fortifikation Studies 2, 2016, p. 645-661.

Anna Philippa-Touchais, « Death in the early Middle Helladic period (MH I-II): Diversity in the construction of mnemonic landscapes ", in Elisabetta Borgna, Ilaria Caloi, Filippo Carinci, Robert Laffineur (éd.), MNHMH/MNEME. Past and memory in the Aegean Bronze Age, Leuven, Peeters, 2019, p. 221-231.

Anna Philippa-Touchais, Gilles Touchais, « Rapport sur les travaux de l'École française d'Athènes en 1995. Argos, Aspis », BCH 120, 1996, p. 843-845.

Anna Philippa-Touchais, Gilles Touchais, « Rapport sur les travaux de l'École française d'Athènes en 1996. Argos, Aspis », BCH 121, 1997, p. 752-753.

Anna Philippa-Touchais, Gilles Touchais, « Les fouilles de l'Aspis d'Argos: la Grèce avant les palais mycéniens », Dossiers d'archéologie 222, 1997, p. 76-81.

Anna Philippa-Touchais, Gilles Touchais, « Rapport sur les travaux de l'École française d'Athènes en 1999. Argos, Aspis », BCH 124, 2000, p. 489.

31 Anna Philippa-Touchais, Gilles Touchais, «Rapport sur les travaux de l'École française d'Athènes en 2000. Argos, 1. Aspis ", BCH 125, 2001, p. 563-564.

Anna Philippa-Touchais, Gilles Touchais, « Rapport sur les travaux de l'École française d'Athènes en 2001. Argos, Aspis », BCH 126, 2002, p. 494-500. 

landscape (MH II-LH II) », in Birgitta Eder, Michaela Zavadil (éd.), (Social) Place and Space in Early Mycenaean Greece. International Discussions in Mycenaean Archaeology, 5th-8th October, 2016 in Athens, Vienne, à paraître.

Anna Philippa-Touchais, Gilles Touchais, « Rapport sur les travaux de l'École française d'Athènes en 2003 et 2004. Argos, D. Colline de l'Aspis. Étude de la céramique et travaux d'aménagement », BCH 128-129, 2004, p. 836-839.

Anna Philippa-Touchais, Gilles Touchais, « Rapport sur les travaux de l'École française d'Athènes en 2005. Argos, Aspis », BCH 130, 2006, p. 714-721.

Anna Philippa-Touchais, Gilles Touchais, « Rapport sur les travaux de l'École française d'Athènes en 2006. Argos, II. Aspis », BCH 131, 2007, p. 960-971.

Anna Philippa-Touchais, Gilles Touchais, « Rapports sur les travaux de l'École française d'Athènes en 2007. Argos. L'Aspis », BCH 132, 2008, p. 767-784.

Anna Philippa-Touchais, Gilles Touchais, «The rise to complexity of a pre-palatial society: The MH settlement of Aspis at Argos », BICS 51, 2008, p. 193-194.

Anna Philippa-Touchais, Gilles Touchais, « Rapports sur les travaux de l'École française d'Athènes en 2008. Argos. L'Aspis », BCH 133, 2009, p. 567-580.

Anna Philippa-Touchais, Gilles Touchais, « Rapports sur les travaux de l'École française d'Athènes en 2010. Argos. La Deiras », BCH 135, 2011, p. 551-566.

Anna Philippa-Touchais, Gilles Touchais, «Fragments of the pottery equipment of an Early Middle Helladic household from Aspis, Argos ", in Walter Gauss, Michael Lindblom, R. Angus K. Smith, James C. Wright (éd.), Our Cups Are Full. Pottery and Society in the Aegean Bronze Age, Papers presented to Jeremy B. Rutter on the Occasion of his 65th birthday, Oxford, Archeopress, 2011, p. 203-216.

Anna Philippa-Touchais, Gilles Touchais, « Rapports sur les travaux de l'École française d'Athènes en 2013. Argos. L'Aspis », BCH 138, 2014, p. 733-749.

Anna Philippa-Touchais, Gilles Touchais, «Travaux de l'École française d'Athènes en 2014 et 2015. Argos. L'Aspis », BCH 139-140, 2015-2016, p. 799-827.

Anna Philippa-Touchais, Gilles Touchais, "Glow in the "Dark" : A gold pendant from a Middle Helladic settlement (Aspis, Argos) », in Jan Driessen (éd.), RA-PI-NE-U. Studies on the Mycenaean World in Honour of Robert Laffineur for His 70th Birthday (Aegis 10), Louvainla-Neuve, PU, 2016, p. 275-293.

Anna Philippa-Touchais, Gilles Touchais, Sofia Voutsaki, James Wright (éd.), Mesohelladika. La Grèce continentale au Bronze Moyen, Athènes, EFA, BCH Suppl. 52, 2010.

Anna Philippa-Touchais et alii, « Rapports sur les travaux de l'École française d'Athènes en 2009. Argos. Aspis », BCH 134, 2010, p. 551-566.

Anna Philippa-Touchais et alii, «II. Rapports 2011. Argos. L'Aspis ; III. Rapports 2012. Argos. L'Aspis », BCH 136-137, p. 593-611 et 799-817.

Anna Philippa-Touchais et alii, « Environnement, alimentation, hygiène et mode de vie dans la Grèce mésohelladique: le cas de l'Aspis d'Argos, in Gilles Touchais, Robert Laffineur, Françoise Rougemont (éd.), PHYSIS. L'environnement naturel et la relation homme-milieu dans le monde égéen protohistorique, Liège, Peeters, Aegaeum 37, 2014, p. 532-538.

Anna Philippa-Touchais et alii, " The social dynamics of Argos in a constantly changing 
Marcel Piérart, Gilles Touchais, Argos, une ville grecque de 6000 ans, Paris, CNRS, 1996.

Gilles Touchais, «Rapports sur les travaux de l'École française en 1974. Argos, IV. Aspis », BCH 99, 1975, p. 707-708.

Gilles Touchais, «Rapports sur les travaux de l'École française en 1975. Argos, III. Aspis », BCH 100, 1976, p. 755-758 et fig. 9 dépliant V (plan K. Kolokotsas).

Gilles Touchais, « Rapports sur les travaux de l'École française en Grèce en 1977. Argos, V. Aspis », BCH 102, 1978, p. 798-802.

Gilles Touchais, « Rapports sur les travaux de l'École française en Grèce en 1979. Argos, III. Aspis », BCH 104, 1980, p. 698-699.

Gilles Touchais, "La céramique néolithique de l'Aspis ", in Études argiennes, Athènes, EFA, BCH Suppl. 6, p. 1-42.

Gilles Touchais, « Rapports sur les travaux de l'École française en Grèce en 1983. Argos, 6. Aspis », BCH 108, 1984, p. 850-852.

Gilles Touchais, « Rapport sur les travaux de l'École française d'Athènes en 1989. Argos, 4. L’Aspis », BCH 114, 1990, p. 872-875.

Gilles Touchais, « Rapport sur les travaux de l'École française d'Athènes en 1990. Argos, 3. L’Aspis », BCH 115, 1991, p. 682-686.

Gilles Touchais, "Argos à l'époque mésohelladique : un habitat ou des habitats? », in Anna Pariente, Gilles Touchais (éd.), Argos et l'Argolide. Topographie et urbanisme, Athènes, EFA, Études franco-helléniques 3, 1998, p. 71-84.

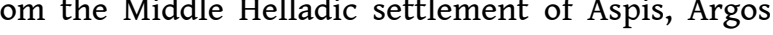
Local production and imports ", in Florens Felten, Walter Gauss, Rudolfine Smetana (éd.), Middle Helladic Pottery and Synchronisms, Vienne, Verlag der Österreichischen Akademie der Wissenschaften, 2007, p. 81-96.

Gilles Touchais, «La colline de l'Aspis à l'époque mycénienne », in Dominique Mulliez (éd.), Sur les pas de Wilhelm Vollgraff. Cent ans d'activités archéologiques à Argos, Athènes, EFA, Recherches franco-helléniques 4, 2013, p. 101-139.

\section{BIBLIOGRAPHIE}

\section{LANGDON 2013}

Susan Langdon, «Children as learners and producers in Early Greece », in Judith Evans Grubbs, Tim Parkin (éd.) The Oxford Handbook of Childhood and Education in the Classical World, Oxford, OUP, 2013, p. 1-19.

TOUCHAIS, FACHARD à paraître

Gilles Touchais, Sylvian Fachard (éd.), Argos, les fouilles de l'Aspis, 1 : Les occupations d'époque historique, Athènes, EFA, Et. pélo. 15, à paraître. 


\section{NOTES}

1. TOUCHAIS, FACHARD à paraitre. L'ouvrage présente l'ensemble des vestiges (architecture et mobilier) liés à l'occupation de la colline depuis l'époque géométrique jusqu'à la fin de l'époque hellénistique. Toute cette documentation provient, pour l'essentiel, des fouilles récentes (1974-2015), mais elle inclut aussi du matériel inédit issu des premières fouilles de Vollgraff (1902-1903).

2. Gilles Touchais et Anne Philippa-Touchais (éds), Argos. Les fouilles de l'Aspis, 2. L'habitat de l'Helladique Moyen (ca 2000-1600 av. J.-C.), en préparation.

3. $B C H$ 139-140, 2015-2016, p. 808. Cet ensemble fera l'objet d'une publication séparée.

4. $B C H$ 138, 2014, p. 738.

5. $B C H$ 31, 1907, p. 156.

6. $B C H 31,1907$, fig. 5, et $B C H 139-140,2015-2016$, p. 809, fig. 5 b.

7. Supra, n. 3.

8. LANGDON 2013.

INDEX

Thèmes : EFA

Année de l'opération : 2017, 2018

peuples https://ark.frantiq.fr/ark:/26678/pcrttls2ZeQkKE

lieux https://ark.frantiq.fr/ark:/26678/pcrt1QBT2dSrXi, https://ark.frantiq.fr/ark:/26678/ pcrts48GOolgr7, https://ark.frantiq.fr/ark:/26678/pcrtRm0r24dwUy sujets https://ark.frantiq.fr/ark:/26678/pcrtSPbJxwzVmX, https://ark.frantiq.fr/ark:/26678/ pcrtBx4xajlijR, https://ark.frantiq.fr/ark:/26678/pcrt1DMOWvDF4j, https://ark.frantiq.fr/ark:/ 26678/pcrtqEWFq9RGda, https://ark.frantiq.fr/ark:/26678/pcrtL7wUJowBSL

\section{AUTEURS}

\section{ANNA PHILIPPA-TOUCHAIS}

UMR 7041 « Archéologies et Sciences de l'Antiquité (ArScAn)»

\section{GILLES TOUCHAIS}

Université Paris 1, UMR 7041 « Archéologies et Sciences de l'Antiquité (ArScAn) »

\section{NATALIE ABELL}

Université de Michigan

\section{ANTHI BALITSARI}

Université de Louvain-la-Neuve 
MARIA CHOLEVA

Fondation Fyssen

JULIE HRUBY

Bryn Mawr College 